\title{
Interview with Richard H Guy
}

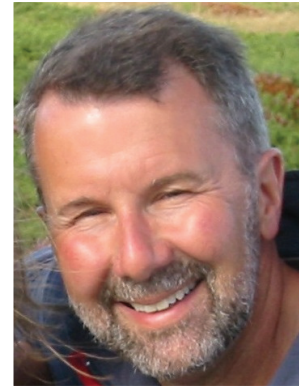

Interview conducted by Hannah Coaker, Assistant Commissioning Editor
Richard Guy received an MA in chemistry from Oxford University (Oxford, UK), and his PhD in pharmaceutical chemistry from the University of London (London, UK). He has held academic posts at the University of California, San Francisco (CA, USA), and the University of Geneva (Geneva, Switzerland). In 2004, he joined the University of Bath (Bath, UK) as Professor of Pharmaceutical Sciences. Professor Guy's research focuses on skin barrier function characterization, transdermal drug delivery, enhancement of percutaneous absorption, iontophoresis, noninvasive biosensing and the prediction and assessment of skin penetration and topical bioavailability.
How did you originally become involved in the field of drug delivery and specifically the field of transdermal/topical administration?

I began by studying chemistry at Oxford University (Oxford, UK). Due to a variety of unusual circumstances, my final year research project involved the mechanism by which compounds penetrated the skin. Even though my research predominantly involved physical chemistry and mathematical modeling, this was where my interest in the skin originated.

I then went to London to do my PhD and began a close collaboration with Jonathan Hadgraft. We first published several theoretical papers about skin absorption, and this work effectively set my career on the skin-delivery path, from which I have never really deviated over the past 30 years.

What are the advantages of delivery via the skin?

Topical delivery is primarily targeted at treating skin disease and therefore has the obvious advantage of applying the formulation directly to the area of skin that is diseased or damaged.

For transdermal drug delivery, the most often cited advantage is that it allows one to deliver compounds that, if given orally, are unavailable - for example, compounds that are subjected to a significant hepatic first-pass effect. Administration via the skin avoids this presystemic metabolism. For example, the dose of estradiol (used in hormone replacement therapy) required transdermally is much less than that when taken orally, due to the avoidance of this metabolic first-pass effect. Another advantage is that drug transport across the skin is slow, permitting delivery to be sustained over a longer period of time, extending the duration of action and thereby reducing the frequency with which the drug needs to be dosed. A further benefit is that inter-subject variability between patients can be reduced if the dosing rate is at least partially controlled by the patch (as opposed to the skin). An additional large advantage is improved patient compliance and acceptability - the patch is now well-accepted, often with less side effects than more conventional oral or injection therapy. Last and not least, transdermal administration is the only route, which I am aware of, where drug input can be stopped if necessary. Although, upon removal of patch, one cannot retrieve drug that has already been absorbed from the patch, it most certainly ensures that no more can enter the patient.

Can you update us on your research group's current work?

Specific ongoing projects include: measurement of the skin's biomechanical properties at the nanoscale using atomic force microscopy; the development and validation of in vitro-in vivo correlations for the assessment of topical drug product bioequivalence; the potential of polymeric filmforming systems as sustained release platforms for topical drugs; determination of the disposition of drug and formulation excipients (including nanoparticles) postapplication to the skin and nail using coherent Raman scattering (CRS) and confocal microscopy; derivation and evaluation of predictive models of percutaneous penetration for pharmaceutical and cosmetic 'actives', and for potentially toxic chemicals, which come into contact with skin.

\author{
Richard H Guy \\ Department of Pharmacy \\ \& Pharmacology, Claverton Down, \\ Bath, BA2 7AY, UK \\ Tel.: +44 1225384901 \\ Fax: +44 I2253861।4 \\ E-mail: r.h.guy@bath.ac.uk
}




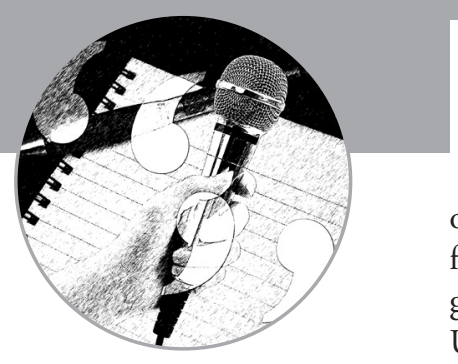

In addition, with respect to noninvasive biosensing using 'reverse' iontophoresis, we are working on models of eczema, attempting to characterize the composition of so-called natural moisturizing factor in diseased skin as compared with healthy skin. We also remain interested in noninvasive glucose monitoring and a collaborative project with colleagues in Chemistry and Physics at the University of Bath (Bath, UK) is examining whether our extraction platform can be interfaced with a graphene-based biosensor device.

Q Is there an aspect of your research that you are particularly excited about at the moment? We have recently been using a new technique CRS to visualize the transport of drugs and vehicle constituents across the skin. CRS is a label-free imaging technique that is capable of real-time, noninvasive examination of living cells and organisms based on molecular vibrational spectroscopy.

Using this approach, we have been able to track the pathways that drugs and excipients from their formulations penetrate across the skin's barrier. It has also been possible to demonstrate that the faster permeation of certain excipients can 'strand' the drug on or near the skin surface in a precipitated form, severely compromising its subsequent bioavailability. This can also occur as volatile excipients (e.g., water, ethanol) evaporate as the formulation is rubbed into the skin. The 'metamorphosis' of the vehicle is a crucial determinant, therefore, of its performance, and much more research needs to be targeted at the residual film left on the skin after this has taken place. With the information provided by CRS, it should be possible to develop and optimize topical formulations that can deliver a much improved 'payload' over those in current use.

Q Can environmental factors, aging or dermatological pathologies significantly affect skin permeability? If so, how is it possible to ensure uniformity in the delivery of a drug via the skin? Yes, these factors can affect the permeability of the skin. Pathologies are more significant; many skin diseases result in changes to skin barrier function (e.g., in eczema, the principal barrier to drug penetration, the stratum corneum, is compromised). A weaker than normal skin barrier will permit greater drug absorption - this is good for therapy, but the risk of systemic exposure is also increased. Of course, resolution of the disease, and recovery of barrier function will also lead to less efficient absorption, but hopefully not before that problem has been resolved.

My personal feeling is that skin barrier function, and drug delivery into and through the skin, are relatively consistent over the age range of $0-100$ years! The major role of the stratum corneum is to keep water inside the body and to impede its passive loss to the atmosphere as much as possible - a task, that it performs admirably well. In maintaining such a good resistance to water transport, the stratum corneum also does a fine job as a permeability barrier to exogenously applied drugs throughout the human life-span.

I can think of only a couple of exceptions. First, very premature infants arrive with an unformed, or rudimentary, stratum corneum and their skin is therefore very permeable. Second, skin which has been burned will also have lost its ability to act as a transport barrier. Other environmental factors may also affect skin barrier function, such as inflammation (due to sun exposure, contact with corrosive chemicals, among others).

Q Is there an opportunity to use CRS to assess and, therefore personalize, delivery for those with dermatological pathologies where the absorption varies more significantly? Right now, CRS is primarily a research tool that is not widely available. We've been lucky enough to perform collaborative projects with Sunney Xie's group at Harvard University (MA, USA) and, more recently, with Julian Moger's laboratory at the University of Exeter (Exeter, UK), where they have the CRS equipment. Whether the research in progress will ultimately have an impact on personalized therapy is hard to predict. What is important is that it should lead to better formulations that perform more efficiently, and that this should be of benefit to all patients requiring therapy applied to their skin.

Q The results of one of your recent publications demonstrate that nanoparticles as formulation components of topical delivery systems do not penetrate further than the superficial layers of skin. What is the significance of this discovery and how do you envisage it will impact the development of future of transdermal and topical formulations?

We found that even the smallest of nanoparticles studied did not penetrate the skin's surface. We used a technique called laser scanning confocal microscopy to examine whether fluorescently 
tagged polystyrene beads, ranging in size from 20 to 200 nanometers, were absorbed into the skin. However, even when the skin sample had been partially compromised by stripping the outer layers with adhesive tape, the nanoparticles did not penetrate the stratum corneum. Thus, despite the fact that previous studies had reached conflicting conclusions over whether nanoparticles can penetrate the skin or not, we were able to unambiguously visualize and objectively assess what happens to nanoparticles on an uneven skin surface. Whereas earlier work had suggested that nanoparticles appear to penetrate the skin, our results indicated that they may in fact have simply been deposited into a deep crease within the skin sample. More positively, the results suggest that it might be possible to design a new type of nanoparticle-based drug formulation that can be applied to the skin and give controlled release of a drug over a long period of time. This would enable sustained delivery of the active drug, potentially reducing the frequency with which the patient would have to apply the formulation to the skin. We have also noticed that nanoparticles appear to have an affinity for hair follicles. With this in mind, there may be an opportunity to treat diseases such as acne more efficiently, if one can figure out how to 'lodge' the therapeutic particle in the hair follicle and then release the drug directly to the required site of action. While the strategy may or may not require a nano-sized object, a successful application would be a potential outcome of our research.

Q Much of your work focuses on enhancing skin permeability for the topical delivery of drugs using sonophoresis and, more recently, iontophoresis. What are the challenges of applying these techniques?

While we have worked a lot on sonophoresis in the past, we have not used the approach since moving to Bath in 2004. The idea of using ultrasound to enhance topical/transdermal drug delivery has been around for some time now, but there is as yet no therapeutic system on the market. The challenge is to marry this technology with a suitable drug, a patient-friendly formulation and the target disease state, where the use of ultrasound satisfies an unmet medical need. So far, this has not been achieved, and I'm not aware of anything on the horizon to make me change this assessment.

Iontophoresis has been around even longer, of course - over a 100 years! In this case, there have been products that have made it to the market with regulatory approval from the US FDA and the European Medicines Agency. Devices have been developed for delivering lidocaine to promote local anesthesia (LidoSite ${ }^{\circledR}$ ), for the administration of fentanyl to treat systemic pain (Ionsys ${ }^{\mathrm{TM}}$ ), and a noninvasive product for monitoring glucose levels in diabetics (GlucoWatch Biographer ${ }^{\mathrm{TM}}$ ). The FDA has also recently approved another iontophoretic device for the delivery of a migraine drug. Unfortunately, none of these products has been a commercial success and none are presently available on the market. The reasons for this lack of return on investment are complex and different for each device. In each case, the apparent benefits of using iontophoresis have been undermined by factors not always related to the use of the technology.

What factors do you think are responsible for preventing the successful commercial application of iontophoresis?

The challenge is identifying the right drug-disease combination for which iontophoresis represents real added-value over and above the existing approach and alternative options. For lidocaine, for example, iontophoresis is able to induce local anesthesia much faster than a typical, topical cream, but the use of the device is more complex, requiring more health professional time to set up and explain to the patient, and it is more expensive. Therefore, at the end of the day, is the shortening of time required for the pharmacological effect to be induced a sufficient benefit to outweigh the less positive features?

There is also a question of how many drugs are really in the 'pool' of potential candidates for iontophoresis. Even though significant enhancement can be achieved with the approach, the fact is that any drug is still going to have to be quite potent (i.e., just as is the case for passive transdermal delivery). In other words, iontophoresis does not expand the number of drugs suitable for administration across the skin by a huge amount. Yes, some peptides (e.g., LHRH or GnRH analogs) enter the picture but the MW limit remains at no more than $20 \mathrm{KDa}$ at the present time. To deliver larger species, or compounds requiring higher doses, necessitates a more aggressive enhancement strategy, such as via the creation of new pathways through the barrier using microneedles, or other poration technologies. 


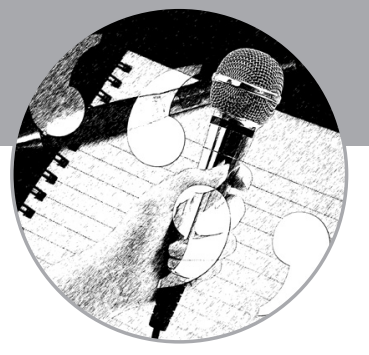

You mentioned that there is an issue with finding the right niche for iontophoresis in the transdermal drug delivery market. In what circumstances would iontophoresis be the technique of choice for enhancing delivery?

A new product that has just been approved delivers the anti-migraine drug, sumatriptan. This is an interesting concept because iontophoresis delivers this potent drug into the body quickly, giving a fast response. Often, migraine sufferers are nauseous, so delivery via the skin is a good idea and being able to control the dose with the current is very desirable - so there is an opportunity here, I believe. Equally, there are some Parkinson's disease drugs that may well be good candidates for iontophoresis. A controlled patch system that self-activates, for example, when the patient wakes up - as people with Parkinson's disease often wake up 'frozen' - could be really beneficial.

Another very promising development program used iontophoresis to deliver gonadotropin-releasing hormone for fertility treatment. Administration of this peptide in the current treatment regimen requires numerous subcutaneous injections to be effective, and the resulting plasma concentration versus time profile can, in fact, be mimicked very closely with iontophoresis applied in an appropriate pulsatile fashion. Unfortunately this project was abandoned, not for reasons relating to the science or the feasibility, even though the results of the initial clinical studies were excellent. In my opinion, this idea needs to be resurrected and developed further.

Do you think that in terms of iontophoresis, the need for more reliable current/delivery profiles poses an obstacle to the broad use of this technique?

No, I do not agree. With iontophoresis different drug input profiles can be provided: with constant current, constant delivery is obtained; with pulsatile current, on and off delivery is possible (see GnRH discussion above); manipulating the current allows the dose delivered to be increased or decreased, such as changing the flow rate on an infusion pump. The microelectronic field is advanced enough to produce devices capable of all of the above. There seems little excuse today not to be able to produce a user-friendly, relatively affordable and discrete delivery system. I do not see this as the limiting factor, which is (as I've said already) addressing the right unmet medical need.

You mentioned that iontophoresis has faced some challenges in terms of becoming an established technique in the medicinal field. Due to the regulatory requirements of drug-device combinations in transdermal delivery, do you anticipate that iontophoresis may first become more successful on the cosmetics market?

Not really. While applications of iontophoresis in the cosmetic world have been explored (and may still be in the process of being examined), there are some clear factors, which limit the likely success of the approach in this regard. First of all, many cosmetics are applied over relatively large areas of skin and the form of an iontophoretic device to achieve such an objective is not obvious. Second, even if one could envisage a system whereby an iontophoretic applicator was moved manually over the skin to be treated, the duration of application would necessarily be short and the quantity of an active that might be delivered in such a limited time would be minimal (and therefore of dubious efficacy). Third, even a small plaster-like system for application around the eyes to diminish crow's feet, for example, would suffer from the same issue pertaining to delivery of a useful amount of the active ingredient.

Q Despite the advantages of transdermal delivery, there are relatively few transdermal drugs that deliver systemic levels of their active ingredient on the market in the EU and USA. What do you think is the reason for this?

Transdermal administration is limited by several factors. Crucially, it is only useful for potent drugs whose daily dose is on the order of $10 \mathrm{mg}$ or (usually) less. Most importantly, higher doses can only be achieved by increasing patch size. In general, practically and aesthetically, $100 \mathrm{~cm}^{2}$ is the maximum acceptable. Transdermal drugs are 'small' molecules, having molecular weights (up until now) of no more than $500 \mathrm{Da}$; lipophilicity is mandatory but, as the drug has to partition into and then exit from the stratum corneum to ultimately become available systemically, a finite aqueous solubility is also required. Logically, transdermal delivery is most suitable for drugs that have low (if any) oral bioavailability (otherwise, why go to the trouble of making a patch?), and short biological half-lives (typically reflecting a significant first-pass effect and a resulting inconvenient dosing regimen). Finally, the drug must not be a skin irritant or sensitizer. 
The following drugs are approved for transdermal delivery: scopolamine (motion sickness), nitroglycerin and isosorbide dinitrate (angina pectoris), clonidine (hypertension), estradiol (hormone replacement therapy), fentanyl (analgesia), nicotine (smoking cessation), testosterone (hypogonadism), norelgestromin + ethinyl estradiol (contraception), oxybutynin (incontinence), selegiline (depression), methylphenidate (attention deficit hyperactivity disorder), buprenorphine (analgesia), rivastigmine (dementia), rotigotine (anti-Parkinsonian) and granisetron (anti-emetic). The range of diseases treated transdermally is broad and the only link common to all these drugs is their potency. Rotigotine represents the first new chemical entity developed from scratch for transdermal delivery. All the rest were previously approved for delivery by more conventional routes (usually oral) before they were developed as transdermals.

I suspect that, in the compound libraries of many pharmaceutical companies, there are many compounds that have been eliminated as drug candidates due to perceived metabolic sensitivity early on in development. Some of these compounds might well be very good candidates for transdermal delivery and could be very effective if given across the skin, but they have fallen by the wayside because companies have not had the mind-set that, if this potent compound cannot be given orally, perhaps it could be administered by a different route. Rotigotine is the unique exception to-date. With luck, its success will spawn other examples and provide the transdermal field with new and interesting opportunities.

Can you expand on the specific properties that would make a drug a strong candidate for transdermal delivery, for example, are transdermally delivered drugs often more lipophilic to ensure penetration of the skin?

Oral drugs are typically categorized using the Biopharmaceutical Classification System (BCS), into class I, II, III and IV drugs. BCS I drugs have good aqueous solubility, dissolve easily and have decent lipophilicity, facilitating their good absorption characteristics at the gut membrane. BCS II drugs are polar with good water solubility, but absorb poorly across the GI membrane. BCS III compounds have poor aqueous solubility, but are lipophilic and permeate the GI membrane well. BCS IV drugs are often relatively insoluble in most solvents, are sometimes of higher molecular weight, and struggle with both dissolution and membrane transport as a result. When drug companies develop new compounds, their BCS classification is quickly determined and those in class IV are left by the wayside, as it is recognized that their administration (especially orally) is going to be very difficult. The idea that you can take such a compound and instead put it on the skin is absurd. The gut has evolved over millions of years to be a good surface for absorbing the nutrients from our diet and the skin has evolved over a similar period of time to keep things out. With this in mind, if a compound is not going to be absorbed through the gut, the chances of it penetrating the skin in any useful therapeutic amount are infinitesimal. Nevertheless, it is not unusual to be asked as a consultant to come up with ways to 'rescue' an completely inappropriate candidate for transdermal delivery!

For oral delivery, it is common these days to apply Lipinski's Rule-of-five when deciding whether or not to consider a candidate further. In fact, this rule works pretty well for the transdermal route too: big molecules ( $>500 \mathrm{Da}$ ) permeate poorly; very lipophilic compounds $(\log \mathrm{P}>5)$ have low transdermal flux rates; very polar compounds, capable of donating or accepting lots of hydrogenbonds, do not partition very well into the lipophilic stratum corneum, and therefore penetrate very slowly as well. This is not exactly rocket science.

Q Do you think that regulatory bodies need to introduce stricter guidelines in terms of risk management and quality control to increase the number of transdermal drugs that are successful at clinical trials?

I do not think that there is a regulatory problem with respect to risk management. In the earlier days of transdermal delivery, a number of compounds made it some way along the development pathway before skin irritation was identified as a significant and insurmountable problem. Today, the evaluation of skin irritation potential is undertaken sooner and unpleasant surprises have been reduced.

Equally, it is not the regulators' fault that false hopes based on frankly crazy ideas (e.g., the passive delivery of vaccines across intact skin, the ability of cell-penetrating peptides to 'magic' all sorts of compounds across a functional barrier, among others) have not borne fruit. 


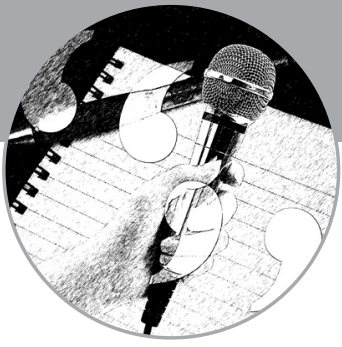

Q How do you anticipate the field of transdermal drug delivery will progress in the next $5-10$ years?

The recent attention on creating new pathways across the skin's barrier, short circuiting the stratum corneum in a minimally invasive fashion, using microneedles, heat poration or laser poration, is of interest and there is much hope that new products will emerge. Vaccination is a logical application, for example using coated microneedles (or dissolvable ones into which the vaccine is incorporated) to take advantage of the skin's local immunological amplification apparatus to provoke the immune response. I expect this approach to come to fruition, but where the field will go next is an interesting question. That is, what is the next unmet medical need that can be addressed with this technology? In what other situations will skin poration provide significant add-on value over a needle and syringe. The latter, after all, is the gold standard for any novel drug delivery approach. It will be interesting to see what's next in the pipeline of applications of these technologies.

I believe that there will be real progress in the development of improved formulations for topical drug delivery, not only for the treatment of dermatological disease, but also for the percutaneous delivery of drugs to relieve subcutaneous inflammation. Recognition of the reasons underlying the presently poor bioavailability of topical drug products must undoubtedly lead to optimized vehicles that deliver a larger payload.

Furthermore, I am optimistic that there will be more 'Rotigotines' in the future, namely that there will be other new drugs being developed ab initio for transdermal delivery, and I am hopeful that iontophoresis products will eventually be commercialized successfully.

What words of advice would you give young scientists envisioning a career in topical/transdermal drug delivery research?

I would say to anyone considering this field of research that you will need a thick skin in the sense that topical/transdermal drug delivery research is a difficult challenge - as I mentioned earlier, the skin is not designed as a surface for drug absorption, it is there to be a barrier, namely, topical/ transdermal drug delivery research is an illogical goal! So, to work in this field requires persistence, stubbornness, a good understanding of the barrier, and an appreciation of the literature. Plenty of mistakes have been made in the past, so there's no need to repeat them!

Finally, I'd emphasize that there remain some important unresolved issues especially with respect to the treatment of skin (and, it should be said, nail) diseases. It should not be forgotten that skin problems result in a substantial number of general practitioner visits and are seen as an important factor in a patient's quality-of-life. The opportunity to have a real impact, therefore, is significant.

\section{Disclaimer}

The opinions expressed in this interview are those of the interviewee and do not necessarily reflect the views of Future Science Ltd.

\section{Financial \& competing interests disclosure}

The interviewee has no relevant affiliations or financial involvement with any organization or entity with a financial interest in or financial conflict with the subject matter or materials discussed in the manuscript. This includes employment, consultancies, honoraria, stock ownership or options, expert testimony, grants or patents received or pending, or royalties.

No writing assistance was utilized in the production of this manuscript. 